\title{
The study of the functional state of endothelium in patients with chronic viral Hepatitis C
}

APASL Single Topic Conference Delta Hepatitis

June 27-28, 2019 Baku, Azerbaijan

Arzu Dadashova ${ }^{1}$

${ }^{1}$ Biochemistry department of Azerbaijan Medical University, Baku, Azerbaijan, arzu26@mail.ru

Keywords: nitric oxide, endothelin-1, chronic viral hepatitis, lipid peroxidation

Introduction Hepatitis $C$ is the leader among hepatotropic infections in developed countries, where $1-24 \%$ of the population is infected. Currently, the pathological mechanisms controlling the rapid progression of the disease are being actively studied and increased sensitivity to oxidative stress may play a role in this. As a result violation of lipid peroxidation (LPO) processes occur. Increased levels of lipid peroxidation contribute to the development of endothelial function, which aggravates liver damage and contributes to the progression of liver fibrosis. Nitric oxide (NO) and endothelin-1 (Et-1) are markers of endothelial dysfunction. The aim of the study. The aim of the study was to determine the level of nitric oxide (NO) and endothelin-1 (Et1 ) in the blood plasma of patients with chronic viral hepatitis $\mathrm{C}(\mathrm{CVHC})$. Blood was examined in 87 patients aged 17-38 years old, which were divided into 2 groups: The I Group - 45 patients with chronic viral hepatitis $\mathrm{C}$ and the II Group - 42 patients with chronic hepatitis C, complicated by bacterial infection (pneumonia). The control group composed of 20 healthy donors. Materials and Methods. The biochemical assessment was carried out by studying markers such as total, direct and indirect bilirubin according to the method of Endrashik; AIAT, AsAT - according to the method of Reitman-Fraenkel, the activity of the enzyme $\mathrm{Y}$-glutamyltransferase - using commercial kits of the company "Diasys"
(Germany). The concentrations of nitric oxide and endothelin-1 were determined by the R \& D system and Cloud Clone Corp. China, respectively. Results and discussion All biochemical parameters (bilirubin, its fractions and ALT activity) increased compared with the norm, and the degree of these changes corresponded to the activity and clinical manifestations of hepatitis. In the II group, the AsAT concentration increased significantly ( $p<0.01)$, and in the I group it was within the normal range. A typical criterion of deterioration and unfavorable prognosis is an increase in the levels of ASAT and AIAT, as well as the enzyme $\mathrm{y}$-glutamyltransferase (in the II group, this index increased by 1.9 times), indicating severe hepatocyte necrobiosis. Markers of the endothelium damage were analyzed in the groups of patients with chronic hepatitis $\mathrm{C}$ and chronic hepatitis $\mathrm{C}$ complicated by pneumonia. Thus, a significant decrease in the level of NO was revealed, which indicates an insufficient basic production of $\mathrm{NO}$ in the endothelium of the patients with chronic viral hepatitits C. In patients of the I group, the content of NO was $18.4 \pm 3.01 \mu \mathrm{mol} / \mathrm{I}$ at a control of $28.32 \pm$ $3.3323 \mu \mathrm{mol} / \mathrm{I}$, whereas in the group of patients with pneumonia, this index decreased to $17.3 \pm 2.98 \mu \mathrm{mol} / \mathrm{I}(p<0.01)$. The concentration of Et-1 in patients increased significantly, indicating an increase in its production in patients with chronic viral 
hepatitits and confirmed the endothelial dysfunction in these patients. In both groups, the concentration of endothelin-1 increased to $5.29 \pm 0.36 \mathrm{pg} / \mathrm{ml}$ and $7.17 \pm 1.16 \mathrm{pg} / \mathrm{ml}$, respectively, at a control of $3.97 \pm 0.28 \mathrm{pg} /$ $\mathrm{ml}$. Conclusion It was revealed that in patients with chronic viral hepatitis C, along with changes in liver function samples and biochemical markers, endothelium dysfunction occurred, which was manifested in the decrease of the nitric oxide production and increase in endothelin-1 synthesis. Increased production of Et-1 and a decrease in NO contribute to the progression of chronic liver damage in patients with chronic viral hepatitis $C$ due to the activation of fibrosis.

"Published on behalf of the Azerbaijan Gastroenterology and Hepatology Association for the APASL STC Hepatitis Delta. All rights reserved. (c) The Author 2019. For permissions please email: editor@ejcs.org or uptodate.az@gmail.com 